\title{
STRONGLY ADEQUATE SETS AND ADDING A CLUB WITH FINITE CONDITIONS
}

\author{
JOHN KRUEGER
}

\begin{abstract}
We continue the study of adequate sets which we began in [2] by introducing the idea of a strongly adequate set, which has an additional requirement on the overlap of two models past their comparison point. We present a forcing poset for adding a club to a fat stationary subset of $\omega_{2}$ with finite conditions, thereby showing that a version of the forcing posets of Friedman [1] and Mitchell [3] for adding a club on $\omega_{2}$ can be developed in the context of adequate sets.
\end{abstract}

The idea of an adequate set of models was introduced by the author in [2] Roughly speaking, an adequate set is a set consisting of countable models which are pairwise membership comparable below a particular ordinal called their comparison point. The relevance of the comparison point is that the two models have only a finite overlap past this ordinal. We presented a general framework in [2] for using adequate sets of models as side conditions in forcing on $\omega_{2}$ with finite conditions. Examples of forcings which fit into this framework include adding a generic function on $\omega_{2}$, forcing a nonreflecting stationary subset of $\omega_{2} \cap \operatorname{cof}(\omega)$, and adding an $\omega_{1}$ Kurepa tree.

In earlier work Friedman [1] and Mitchell [3] separately introduced forcing posets which add a club to a fat stationary subset of $\omega_{2}$ with finite conditions, using countable models as side conditions. In this paper we develop an analogue of these forcings in the context of adequate sets. To achieve this, we introduce the idea of a strongly adequate set of models, which differs from an adequate set by obeying an additional requirement on the overlap of models past their comparison point.

This paper is a sequel to [2]. We assume that the reader is familiar with the material in Sections 1-3 of that paper. Our forcing poset is similar to the FriedmanMitchell posets in the sense that we approximate a generic club using intervals. Neeman's method [4] for adding a club is somewhat different; he adds a generic sequence of models with finite conditions for which the suprema of models appearing on the sequence form a club.

\section{Background Assumptions and Notation}

For easy reference, we review here the notation, concepts, and results of Sections $1-3$ of $[2]$.

Assumption 1: Assume $2^{\omega_{1}}=\omega_{2}$.

2010 Mathematics Subject Classification. 03E40.

Key words and phrases. Adequate sets, strongly adequate sets, models as side conditions, adding a club with finite conditions. 
The reason we make this assumption is because it implies that the elementary substructures of $H\left(\omega_{2}\right)$ which we will use are determined by their set of ordinals. This way we can use sets of ordinals as side conditions instead of models.

Using Assumption 1, fix a bijection $\pi: \omega_{2} \rightarrow H\left(\omega_{2}\right)$. Let $\mathcal{A}$ denote the structure $\left(H\left(\omega_{2}\right), \in, \pi\right)$. The bijection $\pi$ induces a set of definable Skolem functions for $\mathcal{A}$. For any set $a \subseteq H\left(\omega_{2}\right)$, let $S k(a)$ denote the closure of $a$ under some (equivalently any) such set of definable Skolem functions. If $N \prec \mathcal{A}$, then $N=\pi\left[N \cap \omega_{2}\right]=S k\left(N \cap \omega_{2}\right)$. By Lemma 1.4 of [2], if $a$ and $b$ are subsets of $\omega_{2}$ satisfying that $S k(a) \cap \omega_{2}=a$ and $S k(b) \cap \omega_{2}=b$, then $S k(a) \cap S k(b)=S k(a \cap b)$.

Assumption 2: There exists a thin stationary subset of $P_{\omega_{1}}\left(\omega_{2}\right)$.

Fix a stationary set $\mathcal{Y} \subseteq P_{\omega_{1}}\left(\omega_{2}\right)$ which is thin. This means that for all $\beta<\omega_{2}$, $|\{a \cap \beta: a \in \mathcal{Y}\}| \leq \omega_{1}$. Without loss of generality we assume that $\mathcal{Y}$ is closed under initial segments, that is, whenever $a \in \mathcal{Y}$ and $\beta<\omega_{2}$, then $a \cap \beta \in \mathcal{Y}$.

Notation: Let $C$ be the club set of $\beta<\omega_{2}$ such that $S k(\beta) \cap \omega_{2}=\beta$. Let $\Lambda=$ $C \cap \operatorname{cof}\left(\omega_{1}\right)$. Let $\mathcal{X}$ denote the stationary set of $M$ in $\mathcal{Y}$ such that $S k(M) \cap \omega_{2}=M$ and for all $\gamma \in M, \sup (C \cap \gamma) \in M$.

Note that every member of $\Lambda$ is a limit point of $C$.

Suppose that $M$ and $N$ are in $\mathcal{X}$. If $M \in S k(N)$, then $S k(M) \in S k(N)$, $\sup (M) \in N$, and every initial segment of $M$ is in $S k(N)$. Note that if $M \in \mathcal{X}$ and $\beta \in \Lambda$, then $S k(M \cap \beta)=S k(M) \cap S k(\beta)$. It easily follows that if $M \in \mathcal{X}$ and $\beta \in \Lambda$, then $M \cap \beta \in \mathcal{X}$.

The fact that $\mathcal{Y}$ is thin provides the following important consequence: if $M$ is a subset of $\beta$ in $\mathcal{X}$, where $\beta \in \Lambda$, then $M \in S k(\beta)$ (Proposition 1.11 [2]). In particular, if $M \in \mathcal{X}$ and $\beta \in \Lambda$, then $M \cap \beta \in S k(\beta)$.

We now discuss the comparison point $\beta_{M, N}$ of sets $M$ and $N$ in $\mathcal{X}$. The definition of $\beta_{M, N}$ is made relative to a stationary subset of $\Lambda$.

Notation: Fix a stationary set $\Gamma \subseteq \Lambda$.

For a set $M \in \mathcal{X}$, let $\Gamma_{M}$ denote the set of $\beta \in \Gamma$ such that

$$
\beta=\min (\Gamma \backslash(\sup (M \cap \beta))) \text {. }
$$

In other words, $\beta \in \Gamma_{M}$ iff $\beta \in \Gamma$ and $\Gamma \cap[\sup (M \cap \beta), \beta)=\emptyset$. If $\beta \in \Gamma_{M}$, then $\beta$ is the least element of $\Gamma$ which is strictly larger than $\sup (M \cap \beta)$. Note that if $M \subseteq N$, then $\Gamma_{M} \subseteq \Gamma_{N}$.

If $M$ and $N$ are in $\mathcal{X}$, then $\Gamma_{M} \cap \Gamma_{N}$ has a largest element. We sketch a proof of this fact as follows. Note that the first element of $\Gamma$ is in both $\Gamma_{M}$ and $\Gamma_{N}$. If $\Gamma_{M} \cap \Gamma_{N}$ does not have a maximum element, then let $\gamma$ be the supremum of $\Gamma_{M} \cap \Gamma_{N}$. One can then show that $\gamma$ is a limit point of both $M$ and $N$. But then the least element of $\Gamma$ above $\gamma$ is in $\Gamma_{M} \cap \Gamma_{N}$, which is a contradiction. (See Lemma 2.4 of $[2]$.)

Notation: For $M$ and $N$ in $\mathcal{X}$, let $\beta_{M, N}$ denote the maximum element of $\Gamma_{M} \cap \Gamma_{N}$.

One of the most important facts about the comparison point $\beta_{M, N}$ is that $M$ and $N$ share no common elements or limit points above $\beta_{M, N}$. In other words, let 
$M^{\prime}=M \cup \lim (M)$ and $N^{\prime}=N \cup \lim (N)$. Then $M^{\prime} \cap N^{\prime} \subseteq \beta_{M, N}$. The idea of the proof is that if $\xi$ is in $M^{\prime} \cap N^{\prime}$, then the least element of $\Gamma$ above $\xi$ is in $\Gamma_{M} \cap \Gamma_{N}$, and hence less than or equal to $\beta_{M, N}$. (See Proposition 2.6 of [2].)

Definition: Let $A$ be a subset of $\mathcal{X}$. We say that $A$ is adequate if for all $M$ and $N$ in $A$, either $M \cap \beta_{M, N} \in S k(N), N \cap \beta_{M, N} \in S k(M)$, or $M \cap \beta_{M, N}=N \cap \beta_{M, N}$.

Note that a set $A$ is adequate iff for all $M$ and $N$ in $A,\{M, N\}$ is adequate. If $\{M, N\}$ is adequate, then the type of comparison which holds between $M$ and $N$ is determined by $M \cap \omega_{1}$ and $N \cap \omega_{1}$. Namely, since $\omega_{1} \leq \beta_{M, N}, M \cap \beta_{M, N} \in S k(N)$ implies that $M \cap \omega_{1} \in S k(N)$ and hence $M \cap \omega_{1}<N \cap \omega_{1}$. And if $M \cap \beta_{M, N}=$ $N \cap \beta_{M, N}$, intersecting this equation by $\omega_{1}$ yields $M \cap \omega_{1}=N \cap \omega_{1}$. If $A$ is adequate, then an $\in$-minimal element of $A$ is a set $M \in A$ such that $M \cap \omega_{1}$ is minimal. If $M \in A$ is $\in$-minimal, then for all $N \in A$, either $M \cap \beta_{M, N} \in S k(N)$ or $M \cap \beta_{M, N}=N \cap \beta_{M, N}$.

Suppose $\{M, N\}$ is adequate. The remainder sets $R_{N}(M)$ and $R_{M}(N)$ describe the overlap of $M$ and $N$ above their comparison point. Namely, let $R_{M}(N)$ be the set of $\beta$ satisfying either

(1) there is $\gamma \geq \beta_{M, N}$ in $M$ such that $\beta=\min (N \backslash \gamma)$, or

(2) $N \cap \beta_{M, N}$ is either equal to $M \cap \beta_{M, N}$ or is in $S k(M)$, and $\beta=\min (N \backslash$ $\left.\beta_{M, N}\right)$.

The remainder set $R_{M}(N)$ is always finite. For if $R_{M}(N)$ were infinite, then there is an increasing sequence $\left\langle\xi_{n}: n<\omega\right\rangle$ such that $\xi_{n} \in N$ if $n$ is even, and $\xi_{n} \in M$ if $n$ is odd. But then letting $\gamma=\sup _{n} \xi_{n}, \gamma$ is a common limit point of $M$ and $N$ above their comparison point $\beta_{M, N}$, which is impossible. (See Proposition 2.9 of [2].)

\section{Strongly Adequate Sets}

In Proposition 2.12 of [2] we proved that assuming that $\Gamma=\Lambda$, for any adequate pair $\{M, N\}$, the remainder set $R_{N}(M)$ is always a subset of $\Gamma$. This property was needed for showing that the forcing poset we defined for adding a generic function on $\omega_{2}$ preserves cardinals, but it was not needed for the other applications.

In Section 4 below we define a forcing poset for adding a club to a fat stationary subset of $\omega_{2}$ with finite conditions. The arguments we give require both that $\Gamma$ is a proper subset of $\Lambda$, and that the remainder sets are contained in $\Gamma$. This motivates the definition of a strongly adequate set.

Definition 2.1. Let $A \subseteq \mathcal{X}$. Then $A$ is strongly adequate if $A$ is adequate and for all $M$ and $N$ in $A, R_{M}(N) \subseteq \Gamma$.

In Section 3 of [2] we developed some results for combining adequate sets to produce new adequate sets. These results are important for amalgamating conditions over elementary substructures, which is used for preserving cardinals. In this section we show how to adapt those arguments to the case of strongly adequate sets.

By Lemma 3.3 of [2], if $\{M, N\}$ is adequate and $\beta \in \Gamma$, then $\{M \cap \beta, N\}$ is adequate. Note that $M \cap \beta$ and $N$ must compare the same way as do $M$ and $N$, since their type of comparison is determined by their intersection with $\omega_{1}$. 
Let $A$ be adequate and $N \in \mathcal{X}$. Then $A$ is $N$-closed if for all $M \in A$, if $M \cap \beta_{M, N} \in S k(N)$ then $M \cap \beta_{M, N} \in A$. The first goal of this section is to prove that the $N$-closure of a strongly adequate set $A$ with $N \in A$ is strongly adequate. First we prove several technical lemmas.

Lemma 2.2. Let $M$ and $N$ be in $\mathcal{X}$ and let $\beta \in \Gamma$. If $\beta_{M, N} \leq \beta$, then $\beta_{M, N}=$ $\beta_{M \cap \beta, N}$.

Proof. Since $\beta_{M, N} \leq \beta, \sup \left((M \cap \beta) \cap \beta_{M, N}\right)=\sup \left(M \cap \beta_{M, N}\right) ; \operatorname{so} \min (\Gamma \backslash \sup ((M \cap$ $\left.\left.\beta) \cap \beta_{M, N}\right)\right)=\min \left(\Gamma \backslash \sup \left(M \cap \beta_{M, N}\right)\right)=\beta_{M, N}$. Therefore $\beta_{M, N} \in \Gamma_{M \cap \beta}$. It follows that $\beta_{M, N}$ is the largest ordinal in $\Gamma_{M \cap \beta} \cap \Gamma_{N}$, since it is the largest ordinal in $\Gamma_{M} \cap \Gamma_{N}$ and $\Gamma_{M \cap \beta} \cap \Gamma_{N} \subseteq \Gamma_{M} \cap \Gamma_{N}$. So $\beta_{M, N}=\beta_{M \cap \beta, N}$.

Lemma 2.3. Let $\{M, K\}$ be strongly adequate and $\beta \in \Gamma$. If $\beta_{M, K} \leq \beta$, then $\{M \cap \beta, K\}$ is strongly adequate.

Proof. As noted above, $\{M \cap \beta, K\}$ is adequate, and $M \cap \beta$ and $K$ compare the same way as do $M$ and $K$. Since $\beta_{M, K} \leq \beta$, by Lemma 2.2 we have that $\beta_{M, K}=\beta_{M \cap \beta, K}$. Note that $\min \left((M \cap \beta) \backslash \beta_{M \cap \beta, K}\right)$, if it exists, is equal to $\min \left(M \backslash \beta_{M, K}\right)$. So if $(M \cap \beta) \cap \beta_{M \cap \beta, K}$ is either equal to $K \cap \beta_{M \cap \beta, K}$ or is in $S k(K)$, and this minimum exists, then the minimum is in $\Gamma$ since $\{M, K\}$ is strongly adequate. On the other hand, if $K \cap \beta_{M \cap \beta, K}$ is either equal to $(M \cap \beta) \cap \beta_{M \cap \beta, K}$ or is in $S k(M \cap \beta)$, and $\min \left(K \backslash \beta_{M \cap \beta, K}\right)$ exists, then this minimum is equal to $\min \left(K \backslash \beta_{M, K}\right)$ and hence is in $\Gamma$ since $\{M, K\}$ is strongly adequate. Similarly, if $\gamma \in(M \cap \beta) \backslash \beta_{M \cap \beta, K}$, then $\gamma \in M \backslash \beta_{M, K}$; so $\min (K \backslash \gamma)$ is in $\Gamma$ if it exists. And if $\gamma \in K \backslash \beta_{M \cap \beta, K}$, then $\gamma \in K \backslash \beta_{M, K}$; so if $\min ((M \cap \beta) \backslash \gamma)$ exists, then it is equal to $\min (M \backslash \gamma)$ and hence is in $\Gamma$.

Lemma 2.4. Let $\{M, K\}$ be strongly adequate and $\beta \in \Gamma$. If $\beta<\beta_{M, K}$ and $M \cap \beta_{M, K} \in S k(K)$, then $\{M \cap \beta, K\}$ is strongly adequate.

Proof. Note that $M \cap \beta$ is in $S k(K)$ since it is an initial segment of $M \cap \beta_{M, K}$. So clearly $\beta_{M \cap \beta, K}$ is the least element of $\Gamma$ greater than $\sup (M \cap \beta)$. But then both $R_{K}(M \cap \beta)$ and $R_{M \cap \beta}(K)$ are empty.

Proposition 2.5. Let $A$ be strongly adequate and let $N \in A$. Then

$$
A \cup\left\{M \cap \beta_{M, N}: M \in A, M \cap \beta_{M, N} \in S k(N)\right\}
$$

is strongly adequate and $N$-closed.

Proof. If suffices to prove the claim that if $A$ is strongly adequate, $M$ and $N$ are in $A$, and $M \cap \beta_{M, N} \in S k(N)$, then $A \cup\left\{M \cap \beta_{M, N}\right\}$ is strongly adequate. For then any two models in the set described in the lemma will compare properly by two applications of this claim. So let $K \in A$ be given, and we will show that $\left\{M \cap \beta_{M, N}, K\right\}$ is strongly adequate. By the previous two lemmas, it suffices to consider the case when $\beta_{M, N}<\beta_{M, K}$ and $K \cap \beta_{M, K}$ is either equal to $M \cap \beta_{M, K}$ or is is in $S k(M)$.

Note that $K \cap\left[\beta_{M, N}, \beta_{M, K}\right)$ is nonempty. For otherwise $\sup \left(K \cap \beta_{M, K}\right)=$ $\sup \left(K \cap \beta_{M, N}\right)$. But then $\beta_{M, K}=\min \left(\Gamma \backslash \sup \left(K \cap \beta_{M, K}\right)\right)=\min (\Gamma \backslash \sup (K \cap$ $\left.\left.\beta_{M, N}\right)\right) \leq \beta_{M, N}$, which contradicts that $\beta_{M, N}<\beta_{M, K}$.

Suppose that $K \cap \beta_{M, K}=M \cap \beta_{M, K}$. We claim that $\beta_{M \cap \beta_{M, N}, K}=\beta_{M, N}$. Since $\beta_{M, N}<\beta_{M, K}, M \cap \beta_{M, N}=K \cap \beta_{M, N}$. It follows that $\beta_{M \cap \beta_{M, N}, K}$ is the least ordinal in $\Gamma$ above $\sup \left(M \cap \beta_{M, N}\right)$; for this latter ordinal is clearly in $\Gamma_{M \cap \beta_{M, N}} \cap \Gamma_{K}$, 
and it is the largest ordinal in $\Gamma_{M \cap \beta_{M, N}}$. But since $\beta_{M, N} \in \Gamma_{M}$, by definition the least ordinal in $\Gamma$ above $\sup \left(M \cap \beta_{M, N}\right)$ is $\beta_{M, N}$. So $\beta_{M \cap \beta_{M, N}, K}=\beta_{M, N}$. Since obviously there is nothing in $M \cap \beta_{M, N}$ above $\beta_{M, N}, R_{K}\left(M \cap \beta_{M, N}\right)=\emptyset$ and $R_{M \cap \beta_{M, N}}(K)$ is either empty or equal to $\left\{\min \left(K \backslash \beta_{M, N}\right)\right\}$.

Suppose that $\min \left(K \backslash \beta_{M, N}\right)$ exists, and we will prove that it is in $\Gamma$. Recall that $K \cap\left[\beta_{M, N}, \beta_{M, K}\right)$ is nonempty. Since $M \cap \beta_{M, K}=K \cap \beta_{M, K}$, it follows that $\min \left(K \backslash \beta_{M, N}\right)$ is equal to $\min \left(M \backslash \beta_{M, N}\right)$, and this ordinal is in $R_{N}(M)$ and hence in $\Gamma$.

Now assume that $K \cap \beta_{M, K} \in S k(M)$. Then $K \cap \beta_{M, N}$ is an initial segment of $K \cap \beta_{M, K}$ and so is in $S k(M)$; it is also in $S k\left(\beta_{M, N}\right)$. Thus $K \cap \beta_{M, N} \in S k(M) \cap$ $S k\left(\beta_{M, N}\right)=S k\left(M \cap \beta_{M, N}\right)$. No larger initial segment of $K$ can be in $S k\left(\beta_{M, N}\right)$ since it would contain ordinals larger than $\beta_{M, N}$. Therefore $\beta_{M \cap \beta_{M, N}, K} \leq \beta_{M, N}$. Indeed, $\beta_{M \cap \beta_{M, N}, K}$ is the least ordinal in $\Gamma$ larger than $\sup \left(K \cap \beta_{M, N}\right)$.

We claim that $\beta_{M \cap \beta_{M, N}, K}=\beta_{N, K}$. First observe that $K \cap \beta_{M, N} \in S k(M \cap$ $\left.\beta_{M, N}\right) \subseteq S k(N)$. Recall that $K \cap\left[\beta_{M, N}, \beta_{M, K}\right)$ is nonempty. Since $K \cap \beta_{M, K} \in$ $S k(M), K \cap\left[\beta_{M, N}, \beta_{M, K}\right)$ is a nonempty subset of $M \backslash \beta_{M, N}$. Hence $K \cap\left[\beta_{M, N}, \beta_{M, K}\right)$ is nonempty and disjoint from $N$. In particular, the least element of $K \cap\left[\beta_{M, N}, \beta_{M, K}\right)$ is not in $N$, yet any element of $K$ below this least element is in $N$. It follows that $\beta_{N, K}=\min \left(\Gamma \backslash \sup \left(K \cap \beta_{M, N}\right)\right)=\beta_{M \cap \beta_{M, N}, K}$.

In particular, if the ordinal $\min \left(K \backslash \beta_{M \cap \beta_{M, N}, K}\right)$ exists, then it is equal to $\min (K \backslash$ $\left.\beta_{N, K}\right)$, which is in $R_{N}(K)$ and hence in $\Gamma$. Let $\gamma$ in $\left(M \cap \beta_{M, N}\right) \backslash \beta_{M \cap \beta_{M, N}, K}$ be given, and suppose that $\xi=\min (K \backslash \gamma)$. Since $M \cap \beta_{M, N} \in S k(N), \gamma$ is in $N \backslash \beta_{N, K}$. Hence $\xi$ is in $R_{N}(K)$ and therefore in $\Gamma$. Finally, assume that $\gamma$ is in $K \backslash \beta_{M \cap \beta_{M, N}, K}$. Then $\gamma$ is not in $M \cap \beta_{M, N}$. Since $K \cap \beta_{M, N}$ is a subset of $M, \gamma$ must be at least $\beta_{M, N}$. But then $\min \left(\left(M \cap \beta_{M, N}\right) \backslash \gamma\right)$ does not exist.

Proposition 2.6. Let $A$ be strongly adequate, $N \in \mathcal{X}$, and suppose that $A \subseteq$ $S k(N)$. Then $A \cup\{N\}$ is strongly adequate.

Proof. Consider $M \in A$. Then $M \in S k(N)$. So clearly $\beta_{M, N}$ is the least member of $\Gamma$ above $\sup (M)$. So $M \cap \beta_{M, N}=M \in S k(N)$, and both $R_{M}(N)$ and $R_{N}(M)$ are empty.

Before we proceed any further, we prove several technical lemmas regarding comparison points which we will need.

Lemma 2.7. Let $M \in \mathcal{X}, \beta \in \Gamma$, and suppose $M \subseteq \beta$. Then $\Gamma_{M} \subseteq \beta+1$. Therefore for all $N \in \mathcal{X}, \beta_{M, N} \leq \beta$.

Proof. Since $M \subseteq \beta, \sup (M)<\beta$. Let $\gamma \in \Gamma_{M}$ be given. Then $\sup (M \cap \gamma) \leq$ $\sup (M)<\beta$. Since $\beta \in \Gamma$ and $\gamma=\min (\Gamma \backslash \sup (M \cap \gamma)), \gamma \leq \beta$. So $\Gamma_{M} \subseteq \beta+1$. In particular, if $N \in \mathcal{X}$, then by definition $\beta_{M, N} \in \Gamma_{M}$, so $\beta_{M, N} \leq \beta$.

Lemma 2.8. Let $K, M, N \in \mathcal{X}$, and suppose that $M \subseteq N$. Then $\beta_{M, K} \leq \beta_{N, K}$.

Proof. Since $M \subseteq N, \Gamma_{M} \subseteq \Gamma_{N}$. So $\Gamma_{M} \cap \Gamma_{K} \subseteq \Gamma_{N} \cap \Gamma_{K}$. Hence $\beta_{M, K}=$ $\max \left(\Gamma_{M} \cap \Gamma_{K}\right) \leq \max \left(\Gamma_{N} \cap \Gamma_{K}\right)=\beta_{N, K}$.

Lemma 2.9. Let $M$ and $N$ be in $\mathcal{X}$ and let $\beta \in \Gamma$. If $N \subseteq \beta$, then $\beta_{M, N}=\beta_{M \cap \beta, N}$.

Proof. By Lemma 2.2, it suffices to show that $\beta_{M, N} \leq \beta$. This follows from Lemma 2.7 and the fact that $N \subseteq \beta$. 
Proposition 2.11 below describes a method for amalgamating strongly adequate sets over countable elementary substructures. This method is used to preserve $\omega_{1}$ in forcing with finite conditions. The situation is a bit more complicated than for adequate sets; a difference is that we now have to consider an auxiliary set $K$ as described in the next proposition.

Note that if $K \in \mathcal{X}$ and $\gamma$ and $\zeta$ are in $K \cap \Gamma$, then $\{K, K \cap \zeta, K \cap \gamma\}$ is strongly adequate.

Proposition 2.10. Suppose that $A$ is strongly adequate and $N \in A$. Let $R(N)=$ $\bigcup\left\{R_{M}(N): M \in A\right\}$. Assume that $K \in \mathcal{X} \cap S k(N)$ satisfies that $A \cap S k(N) \subseteq$ $S k(K)$ and $R(N) \subseteq K$. Then

$$
(A \cap S k(N)) \cup\{K\} \cup\{K \cap \zeta: \zeta \in R(N)\}
$$

is strongly adequate.

Proof. Let $M \in A \cap S k(N)$ be given. Then $M \in S k(K)$, so by Proposition 2.6, $\{M, K\}$ is strongly adequate. Fix $L \in A$ and $\zeta \in R_{L}(N)$, and we will show that $\{M, K \cap \zeta\}$ is strongly adequate. Note that since $M \in S k(K), M \cap \zeta \in$ $S k(K) \cap S k(\zeta)=S k(K \cap \zeta)$. Since $K \cap \zeta \subseteq \zeta$ and $\zeta \in \Gamma, \beta_{M, K \cap \zeta} \leq \zeta$. Hence $M \cap \beta_{M, K \cap \zeta} \in S k(K \cap \zeta)$. Also $M \cap \zeta=M \cap \beta_{M, K \cap \zeta}$, since $M \cap \zeta \subseteq M \cap(K \cap \zeta) \subseteq$ $\beta_{M, K \cap \zeta}$.

We will show that $R_{K \cap \zeta}(M)$ and $R_{M}(K \cap \zeta)$ are subsets of $\Gamma$. If $\gamma \in M \backslash \beta_{M, K \cap \zeta \text {, }}$ then since $M \cap \zeta=M \cap \beta_{M, K \cap \zeta}, \gamma \geq \zeta$. Hence $\min ((K \cap \zeta) \backslash \gamma)$ does not exist. It follows that $R_{M}(K \cap \zeta)$ is empty.

Now we show that $R_{K \cap \zeta}(M)$ is a subset of $\Gamma$. If $\min \left(M \backslash \beta_{M, K \cap \zeta}\right)$ does not exist, then $R_{K \cap \zeta}(M)$ is the emptyset, so assume that it does exist. Since $M \cap \zeta=$ $M \cap \beta_{M, K \cap \zeta}, \min \left(M \backslash \beta_{M, K \cap \zeta}\right)$ is equal to $\min (M \backslash \zeta)$. On the other hand, if $\gamma \in(K \cap \zeta) \backslash \beta_{M, K \cap \zeta}$, then for the same reason, $\min (M \backslash \gamma)$ is equal to $\min (M \backslash \zeta)$. Therefore letting $\xi=\min (M \backslash \zeta), R_{K \cap \zeta}(M)=\{\xi\}$.

It remains to prove that $\xi=\min (M \backslash \gamma)$ is in $\Gamma$. We will show that $\xi$ is in $R_{L}(M)$, which suffices. Since $M \subseteq N$ and $\zeta \in R_{L}(N), \beta_{M, L} \leq \beta_{N, L} \leq \zeta$. If $\zeta=\min (N \backslash \gamma)$ for some $\gamma \in L \backslash \beta_{N, L}$, then obviously $\xi=\min (M \backslash \gamma)$ and $\gamma \in L \backslash \beta_{M, L}$. So $\xi \in R_{L}(M)$ and we are done.

Otherwise either $N \cap \beta_{N, L}=L \cap \beta_{N, L}$ or $N \cap \beta_{N, L} \in S k(L)$ and $\zeta=\min \left(N \backslash \beta_{L, N}\right)$. Clearly then $\xi=\min \left(M \backslash \beta_{L, N}\right)$. So if $M \cap\left[\beta_{M, L}, \beta_{N, L}\right)=\emptyset$, then $\xi=\min \left(M \backslash \beta_{M, L}\right)$ and hence $\xi \in R_{L}(M)$. Otherwise $M \cap\left[\beta_{M, L}, \beta_{N, L}\right) \neq \emptyset$. Let us show that this is impossible. If $\pi$ is in $M \cap\left[\beta_{M, L}, \beta_{N, L}\right)$, then $\pi \in M \backslash L$. So $\pi \in\left(N \cap \beta_{N, L}\right) \backslash L$. This contradicts the comparison of $N$ and $L$ stated at the beginning of the paragraph.

Proposition 2.11. Let $A$ be strongly adequate, $N \in A$, and suppose that $A$ is $N$ closed. Let $R(N)=\bigcup\left\{R_{M}(N): M \in A\right\}$. Fix $K \in S k(N)$ such that $A \cap S k(N) \subseteq$ $K$ and $R(N) \subseteq K$. Suppose that $B$ is strongly adequate and

$$
(A \cap S k(N)) \cup\{K\} \cup\{K \cap \zeta: \zeta \in R(N)\} \subseteq B \subseteq \mathcal{X} \cap S k(N) .
$$

Then $A \cup B$ is strongly adequate.

Proof. Let $M \in A \backslash S k(N)$ and $L \in B$ be given, and we compare $M$ and $L$. Note that since $L \subseteq N, \beta_{L, M} \leq \beta_{M, N}$ by Lemma 2.8. Therefore $\beta_{L, M}=\beta_{L, M \cap \beta_{M, N}}$ by Lemma 2.2 .

Claim: If $\xi$ is in $R_{M}(L)$ and $\xi=\min (L \backslash \zeta)$ for some $\zeta \in R_{M}(N)$, then $\xi$ is in $\Gamma$. 
If $\xi=\zeta$ then we are done. Otherwise $\zeta \notin L$ and $\zeta<\xi$. Let us compare $K$ and $L$. Recall that $\zeta \in K$. So if $\beta_{K, L} \leq \zeta$, then $\xi$ is in $R_{K}(L)$ and hence in $\Gamma$. Otherwise $\zeta<\beta_{K, L}$. Suppose that $\beta_{K, L} \leq \xi$. Since $\zeta \in\left(K \cap \beta_{K, L}\right) \backslash L$, $L \cap \beta_{K, L} \in S k(K)$, since the other types of comparison of $K$ and $L$ are impossible. As $\zeta<\beta_{K, L} \leq \xi=\min (L \backslash \zeta)$, we get that $\xi=\min \left(L \backslash \beta_{K, L}\right)$. So $\xi$ is in $R_{K}(L)$ and hence in $\Gamma$.

Finally, assume that $\xi<\beta_{K, L}$. Again since $\zeta \in\left(K \cap \beta_{K, L}\right) \backslash L, L \cap \beta_{K, L} \in S k(K)$. We compare $L$ and $K \cap \zeta$. We have that $\beta_{K \cap \zeta, L} \leq \zeta<\beta_{K, L}$ by Lemma 2.7. So $L \cap \beta_{K \cap \zeta, L} \in S k(K) \cap S k(\zeta)=S k(K \cap \zeta)$. But $L \cap \beta_{K \cap \zeta, L}=L \cap \zeta$, since $L \cap \zeta \subseteq L \cap \beta_{K, L} \in S k(K)$ implies that any ordinal in $L \cap \zeta$ is in $L \cap(K \cap \zeta)$ and hence in $\beta_{K \cap \zeta, L}$. Therefore $\xi=\min \left(L \backslash \beta_{K \cap \zeta, L}\right)$. So $\xi$ is in $R_{K \cap \zeta}(L)$ and hence in $\Gamma$. This completes the proof of the claim.

Suppose that $N \cap \beta_{M, N}$ is either equal to $M \cap \beta_{M, N}$ or is in $S k(M)$. Since $L \in S k(N)$ and $\beta_{L, M} \leq \beta_{M, N}, L \cap \beta_{L, M} \in S k\left(N \cap \beta_{M, N}\right) \subseteq S k(M)$. First we prove that $R_{M}(L) \subseteq \Gamma$. Let $\xi=\min \left(L \backslash \beta_{L, M}\right)$. Then $\xi \in N \backslash M$, so $\xi \geq \beta_{M, N}$. Let $\zeta=\min \left(N \backslash \beta_{M, N}\right)$, which is in $R_{M}(N)$. Then $\xi=\min (L \backslash \zeta)$; so $\xi \in \Gamma$ by the claim above. Similarly, assume $\gamma \in M \backslash \beta_{L, M}$ and $\xi=\min (L \backslash \gamma)$. Then $\xi \in N \backslash M$, so $\xi \geq \beta_{M, N}$. If $\gamma \geq \beta_{M, N}$, then $\zeta:=\min (N \backslash \gamma)$ is in $R_{M}(N)$, and $\xi=\min (L \backslash \zeta)$; so $\xi \in \Gamma$ by the claim. Otherwise $\beta_{L, M} \leq \gamma<\beta_{M, N}$. Letting $\sigma:=\min \left(N \backslash \beta_{M, N}\right)$, note that $\sigma \in R_{M}(N)$ and $\xi=\min (L \backslash \sigma)$. So again we have that $\xi$ is in $\Gamma$ by the claim.

Now we prove that $R_{L}(M) \subseteq \Gamma$. Let $\gamma \in L \backslash \beta_{L, M}$ be given and let $\xi=\min (M \backslash \gamma)$. If $\gamma \geq \beta_{M, N}$, then $\gamma \in N \backslash \beta_{M, N}$, so $\xi$ is in $R_{N}(M)$ and hence in $\Gamma$. Otherwise $\beta_{L, M} \leq \gamma<\beta_{M, N}$. Then $\gamma$ is in $N \cap \beta_{M, N}$ and hence in $M$. But this is impossible since then $\gamma \in M \cap L$ and $M \cap L$ is a subset of $\beta_{L, M}$.

It remains to consider the case when $M \cap \beta_{M, N} \in S k(N)$. Since $A$ is $N$-closed, $M \cap \beta_{M, N}$ is in $S k(N)$ and hence in $B$. As $M \cap \beta_{M, N}$ and $L$ are both in $B$, $\left\{M \cap \beta_{M, N}, L\right\}$ is strongly adequate. Recall that $\beta_{L, M}=\beta_{L, M \cap \beta_{M, N}}$. Let $\beta=\beta_{L, M}$.

Suppose that $\left(M \cap \beta_{M, N}\right) \cap \beta$ is either equal to $L \cap \beta$ or is in $S k(L)$. Since $\beta \leq \beta_{M, N}, M \cap \beta_{L, M}=\left(M \cap \beta_{M, N}\right) \cap \beta$ is either equal to $L \cap \beta_{L, M}$ or is in $S k(L)$. Let us show that $R_{L}(M) \subseteq \Gamma$. Suppose that $\xi$ is equal to either $\min (M \backslash \beta)$, or to $\min (M \backslash \gamma)$ for some $\gamma \in L \backslash \beta$. If $\xi<\beta_{M, N}$, then $\xi$ is equal to either $\min \left(\left(M \cap \beta_{M, N}\right) \backslash \beta\right)$, or to $\min \left(\left(M \cap \beta_{M, N}\right) \backslash \gamma\right)$ for some $\gamma \in L \backslash \beta$; in either case, $\xi$ is in $R_{L}\left(M \cap \beta_{M, N}\right)$ and hence in $\Gamma$. On the other hand suppose that $\xi \geq \beta_{M, N}$. If $\xi=\min (M \backslash \beta)$, then clearly $\xi=\min \left(M \backslash \beta_{M, N}\right)$ and hence is in $R_{N}(M)$ and therefore in $\Gamma$. Suppose that $\xi=\min (M \backslash \gamma)$, where $\gamma \in L \backslash \beta$. If $\gamma \leq \beta_{M, N}$, then $\xi=\min \left(M \backslash \beta_{M, N}\right)$ and hence is in $\Gamma$. If $\gamma>\beta_{M, N}$, then since $\gamma$ is in $N \backslash \beta_{M, N}$, $\xi=\min (M \backslash \gamma)$ is in $R_{N}(M)$ and hence in $\Gamma$.

Now we show that $R_{M}(L) \subseteq \Gamma$. Suppose that $\gamma \in M \backslash \beta$, and let $\xi=\min (L \backslash \gamma)$. If $\gamma<\beta_{M, N}$, then $\gamma \in\left(M \cap \beta_{M, N}\right) \backslash \beta$, and therefore $\xi$ is in $R_{M \cap \beta_{M, N}}(L)$ and hence in $\Gamma$. Otherwise $\gamma \geq \beta_{M, N}$. Then $\gamma \in M \backslash \beta_{M, N}$. Let $\zeta=\min (N \backslash \gamma)$. Then $\zeta \in R_{M}(N)$ and $\xi=\min (L \backslash \zeta)$, which implies that $\xi \in \Gamma$ by the claim. Finally, assume that $M \cap \beta$ is equal to $L \cap \beta$, and let $\xi=\min (L \backslash \beta)$. Then $\xi$ is equal to $\min \left(L \backslash \beta_{L, M \cap \beta_{M, N}}\right)$, and so is in $R_{M \cap \beta_{M, N}}(L)$ and hence in $\Gamma$.

In the last remaining case, assume that $L \cap \beta \in S k\left(M \cap \beta_{M, N}\right)$. Then $L \cap \beta \in$ $S k(M)$. Let $\xi=\min (L \backslash \beta)$. Then $\xi$ is in $R_{M \cap \beta_{M, N}}(L)$ and hence in $\Gamma$. Assume that $\gamma \in M \backslash \beta$ and $\xi=\min (L \backslash \gamma)$. If $\gamma \in M \cap \beta_{M, N}$, then $\xi$ is in $R_{M \cap \beta_{M, N}}(L)$ and hence in $\Gamma$. Otherwise $\gamma \geq \beta_{M, N}$. Then $\zeta:=\min (N \backslash \gamma)$ is in $R_{M}(N)$ and 
$\xi=\min (L \backslash \zeta)$; so $\xi \in \Gamma$ by the claim. Now suppose that $\gamma \in L \backslash \beta$, and let $\xi=\min (M \backslash \gamma)$. If $\xi<\beta_{M, N}$, then $\xi=\min \left(\left(M \cap \beta_{M, N}\right) \backslash \gamma\right)$, and hence $\xi$ is in $R_{L}\left(M \cap \beta_{M, N}\right)$ and therefore in $\Gamma$. Suppose $\xi \geq \beta_{M, N}$. If $\gamma \geq \beta_{M, N}$, then $\xi$ is in $R_{N}(M)$ and hence in $\Gamma$. If $\gamma<\beta_{M, N}$, then $\xi=\min \left(M \backslash \beta_{M, N}\right)$; so $\xi$ is in $R_{N}(M)$ and hence in $\Gamma$.

We now turn to the topic of amalgamating strongly adequate sets over models of size $\omega_{1}$. This kind of amalgamation, which is described in Proposition 2.15 below, is used to preserve $\omega_{2}$ in forcing with finite conditions.

Lemma 2.12. Let $A$ be strongly adequate, $\beta \in \Gamma$, and $K \in \mathcal{X}$ with $\beta \in K$. If $A \subseteq S k(K \cap \beta)$, then $A \cup\{K\} \cup\{K \cap \beta\}$ is strongly adequate.

This follows from Proposition 2.6.

Lemma 2.13. Let $A$ be strongly adequate, $\beta \in \Gamma$, and suppose that there is $K \in A$ such that $\beta \in K$ and $K \cap \beta \in A$. Then for all $N \in A$ with $N \backslash \beta \neq \emptyset$, the ordinal $\min (N \backslash \beta)$ is in $\Gamma$.

Proof. Let $N \in A$ and suppose that $\min (N \backslash \beta)$ exists. If $\min (N \backslash \beta)=\beta$ then we are done. Suppose that $\min (N \backslash \beta)>\beta$. If $\beta_{N, K} \leq \beta$, then since $\beta \in K, \min (N \backslash \beta)$ is in $R_{K}(N)$ and hence in $\Gamma$. Assume that $\beta<\beta_{N, K}$. Then since $\beta \in K \backslash N$, we must have that $N \cap \beta_{N, K} \in S k(K)$. Since $\beta_{N, K \cap \beta} \leq \beta<\beta_{N, K}$ by Lemma 2.7, $N \cap \beta_{N, K \cap \beta} \in S k(K) \cap S k(\beta)=S k(K \cap \beta)$. Now $N \cap \beta=\left(N \cap \beta_{N, K}\right) \cap \beta \subseteq$ $N \cap(K \cap \beta) \subseteq \beta_{N, K \cap \beta}$. So $\min (N \backslash \beta)=\min \left(N \backslash \beta_{N, K \cap \beta}\right)$, which is in $R_{K \cap \beta}(N)$ and hence in $\Gamma$.

For an adequate set $A$ and $\beta \in \Gamma$, the set $A$ is $\beta$-closed if for all $M \in A$, $M \cap \beta \in A$. The next proposition says that the $\beta$-closure of a strongly adequate set is strongly adequate.

Proposition 2.14. Let $A$ be strongly adequate, $\beta \in \Gamma$, and suppose that there is $K \in A$ such that $\beta \in K$ and $K \cap \beta \in A$. Then

$$
A \cup\{M \cap \beta: M \in A\}
$$

is strongly adequate and $\beta$-closed.

Proof. It suffices to prove the claim that under the assumptions above, if $M \in A$ then $A \cup\{M \cap \beta\}$ is strongly adequate. For then the statement of the proposition can be proved with two applications of this claim. Fix $M$ and $N$ in $A$, and we will show that $\{M \cap \beta, N\}$ is strongly adequate. By Lemmas 2.3 and 2.4, it suffices to consider the case when $\beta<\beta_{M, N}$ and $N \cap \beta_{M, N}$ is either equal to $M \cap \beta_{M, N}$ or is in $S k(M)$.

Suppose that $N \cap \beta_{M, N}=M \cap \beta_{M, N}$. Then $M \cap \beta=N \cap \beta$. Since $\beta_{M \cap \beta, N} \leq \beta$, $(M \cap \beta) \cap \beta_{M \cap \beta, N}=N \cap \beta_{M \cap \beta, N}$. Also $M \cap \beta=M \cap \beta_{M \cap \beta, N}$, since any ordinal in the set on the left is in both $M \cap \beta$ and $N$, and hence in $\beta_{M \cap \beta, N}$. It follows that $R_{N}(M \cap \beta)$ is empty and $R_{M \cap \beta}(N)$ is either empty or equal to $\left\{\min \left(N \backslash \beta_{M \cap \beta, N}\right)\right\}$. But since $N \cap \beta=M \cap \beta=M \cap \beta_{M \cap \beta, N}$, in the latter case $\min \left(N \backslash \beta_{M \cap \beta, N}\right)=$ $\min (N \backslash \beta)$, which is in $\Gamma$ by Lemma 2.13 .

Now assume that $N \cap \beta_{M, N} \in S k(M)$. Since $\beta<\beta_{M, N}$, it follows that $N \cap \beta \in$ $S k(M) \cap S k(\beta)=S k(M \cap \beta)$. Since $\beta_{M \cap \beta, N} \leq \beta, N \cap \beta_{M \cap \beta, N} \in S k(M \cap \beta)$. Also as $N \cap \beta \subseteq M \cap \beta, N \cap \beta=N \cap \beta_{M \cap \beta, N}$, since any ordinal in the set on the left is in $(M \cap \beta) \cap N$ and hence in $\beta_{M \cap \beta, N}$. So $N \cap\left[\beta_{M \cap \beta, N}, \beta\right)=\emptyset$. It follows that 
$\min \left(N \backslash \beta_{M \cap \beta, N}\right)$, if it exists, is equal to $\min (N \backslash \beta)$, which is in $\Gamma$ by Lemma 2.13. And if $\gamma \in(M \cap \beta) \backslash \beta_{M \cap \beta, N}$ and $\xi=\min (N \backslash \gamma)$, then again $\xi=\min (N \backslash \beta)$, which is in $\Gamma$. Finally, suppose $\gamma \in N \backslash \beta_{M \cap \beta, N}$. Then $\gamma \geq \beta$, so $\min ((M \cap \beta) \backslash \gamma)$ does not exist.

Proposition 2.15. Let $A$ be strongly adequate, $\beta \in \Gamma$, and suppose that $A$ is $\beta$ closed. Assume that there is $K \in A$ such that $\beta \in K$. Let $B$ be a strongly adequate set satisfying

$$
A \cap S k(\beta) \subseteq B \subseteq S k(\beta) .
$$

Then $A \cup B$ is strongly adequate.

Proof. Consider $M \in A \backslash S k(\beta)$ and $N \in B$, and we will prove that $\{M, N\}$ is strongly adequate. Since $A$ is $\beta$-closed, $M \cap \beta \in A$. As we discussed in Section 1 , the thinness of the stationary set $\mathcal{Y}$ implies that $M \cap \beta$ is in $S k(\beta)$. So $M \cap \beta$ is in $A \cap S k(\beta)$ and hence in $B$. Therefore $\{M \cap \beta, N\}$ is strongly adequate. If $M=M \cap \beta$ then we are done. So assume that $M \backslash \beta \neq \emptyset$. Then by Lemma 2.13, the ordinal $\min (M \backslash \beta)$ is in $\Gamma$. Since $N \subseteq \beta, \beta_{M, N} \leq \beta$ by Lemma 2.7. So by Lemma $2.2, \beta_{M, N}=\beta_{M \cap \beta, N}$.

We claim that $M$ and $N$ compare the same way as do $M \cap \beta$ and $N$. If $(M \cap \beta) \cap$ $\beta_{M \cap \beta, N}=N \cap \beta_{M \cap \beta, N}$, then $(M \cap \beta) \cap \beta_{M, N}=M \cap \beta_{M, N}$ is equal to $N \cap \beta_{M, N}$. Suppose $(M \cap \beta) \cap \beta_{M \cap \beta, N} \in S k(N)$. Then $(M \cap \beta) \cap \beta_{M \cap \beta, N}=M \cap \beta_{M, N} \in S k(N)$. Finally, if $N \cap \beta_{M \cap \beta, N} \in S k(M \cap \beta)$, then $N \cap \beta_{M, N} \in S k(M)$.

Now we prove that (1) any ordinal in $R_{N}(M)$ is either in $R_{N}(M \cap \beta)$ or is equal to $\min (M \backslash \beta)$, and $(2) R_{M}(N) \subseteq R_{M \cap \beta}(N)$. It follows that $R_{N}(M)$ and $R_{M}(N)$ are subsets of $\Gamma$.

(1) Note that $\min \left(M \backslash \beta_{M, N}\right)$ is either equal to $\min \left((M \cap \beta) \backslash \beta_{M \cap \beta, N}\right)$, or is equal to $\min (M \backslash \beta)$. So if $M \cap \beta_{M, N}$ is either equal to $N \cap \beta_{M, N}$ or is in $S k(N)$, then (1) is satisfied for $\min \left(M \backslash \beta_{M, N}\right)$. If $\gamma \in N \backslash \beta_{M, N}$, then $\gamma \in N \backslash \beta_{M \cap \beta, N}$. So if $\min (M \backslash \gamma)$ exists, then it is either equal to $\min ((M \cap \beta) \backslash \gamma)$, and hence is in $R_{N}(M \cap \beta)$, or it is equal to $\min (M \backslash \beta)$.

(2) Since $\beta_{M, N}=\beta_{M \cap \beta, N}$, the ordinal $\min \left(N \backslash \beta_{M, N}\right)$ is equal to $\min (N \backslash$ $\beta_{M \cap \beta, N}$ ); so this ordinal is in $R_{M \cap \beta}(N)$ in the case when $N \cap \beta_{M, N}$ is either equal to $M \cap \beta_{M, N}$ or is in $S k(M)$. If $\gamma \in M \backslash \beta_{M, N}$, then either $\gamma \in M \cap \beta$ and $\min (N \backslash \gamma)$ is in $R_{M \cap \beta}(N)$, or $\gamma \geq \beta$ and $\min (N \backslash \gamma)$ does not exist.

\section{Forcing with Strongly Adequate Sets as Side Conditions}

We give an example of a forcing poset with finite conditions which illustrates how the results of the previous section can be used for preserving cardinals.

Definition 3.1. Let $\mathbb{P}$ be the forcing poset whose conditions are finite strongly adequate sets. Let $B \leq A$ if $A \subseteq B$.

Proposition 3.2. The forcing poset $\mathbb{P}$ preserves $\omega_{1}$.

Proof. Let $A \Vdash \dot{g}: \omega \rightarrow \omega_{1}$ is a function. Fix $\theta>\omega_{2}$ regular with $\dot{g} \in H(\theta)$. Let $N^{*}$ be a countable elementary substructure of $H(\theta)$ satisfying that $\mathbb{P}, A, \dot{g}, \pi, \mathcal{X} \in N^{*}$ and $N:=N^{*} \cap \omega_{2} \in \mathcal{X}$. Since $\pi \in N^{*}, N^{*} \cap H\left(\omega_{2}\right)=\pi[N]=S k(N)$. In particular, $N^{*} \cap \mathbb{P} \subseteq S k(N)$.

Let $B=A \cup\{N\}$. Then $B$ is strongly adequate by Proposition 2.6. We will prove that $B$ is $N^{*}$-generic. This implies that $B$ forces that the range of $\dot{g}$ is contained 
in $N$, and therefore $\dot{g}$ does not collapse $\omega_{1}$. Fix a dense set $E \in N^{*}$, and we will show that $N^{*} \cap E$ is predense below $B$.

Let $C \leq B$ be given. Define

$$
D=C \cup\left\{M \cap \beta_{M, N}: M \in C, M \cap \beta_{M, N} \in S k(N)\right\} .
$$

By Proposition 2.5, $D$ is strongly adequate and $N$-closed.

Let $X=N^{*} \cap D$. Since $X$ is a finite subset of $N^{*}, X \in N^{*}$. Let $R(N)=$ $\bigcup\left\{R_{M}(N): M \in D\right\}$. By elementarity we can fix $K \in N^{*} \cap \mathcal{X}$ such that $X \in S k(K)$ and $R(N) \subseteq K$. Let $Y=X \cup\{K\} \cup\{K \cap \zeta: \zeta \in R(N)\}$. By Proposition 2.10, $Y$ is strongly adequate. Since $E$ is dense, we can apply elementarity to fix $Z \leq Y$ in $N^{*} \cap E$. Then by Proposition 2.11, $D \cup Z$ is strongly adequate. So $D \cup Z$ is a condition below $D$ and $Z$, and $Z \in E$. This shows that $N^{*} \cap E$ is predense below $B$.

Note that the condition $B$ in the proof above is actually strongly $N^{*}$-generic (see the comments following Proposition 4.2 in [2]).

Proposition 3.3. The forcing poset $\mathbb{P}$ preserves $\omega_{2}$.

Proof. Let $A \Vdash \dot{g}: \omega_{1} \rightarrow \omega_{2}$ is a function. Fix $\theta>\omega_{2}$ regular such that $\dot{g} \in H(\theta)$. Let $N^{*} \prec H(\theta)$ be of size $\omega_{1}$ such that $\mathbb{P}, A, \dot{g}, \pi, \mathcal{X} \in N^{*}$ and $\beta^{*}:=N^{*} \cap \omega_{2} \in \Gamma$. Since $\pi \in N^{*}, N^{*} \cap H\left(\omega_{2}\right)=\pi\left[\beta^{*}\right]=S k\left(\beta^{*}\right)$. In particular, $N^{*} \cap \mathbb{P} \subseteq S k\left(\beta^{*}\right)$.

Fix $K \in \mathcal{X}$ with $\beta^{*} \in K$ and $A \in S k(K)$. Then $A \in S k(K) \cap S k\left(\beta^{*}\right)=$ $S k\left(K \cap \beta^{*}\right)$. Let $B=A \cup\{K\} \cup\left\{K \cap \beta^{*}\right\}$. Then $B$ is strongly adequate by Lemma 2.12. We claim that $B$ is $N^{*}$-generic. This implies that $B$ forces that the range of $\dot{g}$ is a subset of $N^{*}$, and hence does not collapse $\omega_{2}$. Fix a dense set $E \in N^{*}$, and we show that $N^{*} \cap E$ is predense below $B$.

Let $C \leq B$ be given. Define

$$
D=C \cup\left\{M \cap \beta^{*}: M \in C\right\} .
$$

Then $D$ is strongly adequate and $\beta^{*}$-closed by Proposition 2.14 .

Let $X=N^{*} \cap D$. Then $X$ is in $N^{*}$. Note that $X=S k\left(\beta^{*}\right) \cap D$. Since $E$ is dense, by elementarity we can fix $Y \leq X$ in $N^{*} \cap E$. Then $D \cap S k\left(\beta^{*}\right)=X \subseteq Y \subseteq S k\left(\beta^{*}\right)$.

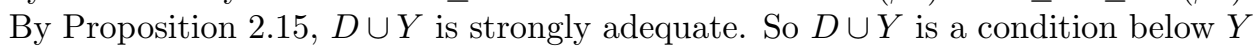
and $D$, and $Y \in N^{*} \cap E$.

Note that since $\mathbb{P}$ has size $\omega_{2}$, it preserves all cardinals larger than $\omega_{2}$ as well.

Let us consider a more complicated example of a forcing poset with strongly adequate sets as side conditions. This example adds a generic function on $\omega_{2}$.

Definition 3.4. Let $\mathbb{P}$ be the forcing poset whose conditions are pairs $(f, A)$ satisfying:

(1) $f$ is a finite partial function from $\omega_{2}$ to $\omega_{2}$;

(2) $A$ is a finite strongly adequate set;

(3) for all $M \in A$ and $\alpha \in \operatorname{dom}(f)$, if $M \cap[\alpha, f(\alpha)] \neq \emptyset$, then $\alpha, f(\alpha) \in M$.

Let $(g, B) \leq(f, A)$ if $A \subseteq B$ and $f \subseteq g$.

A similar forcing poset was defined in Section 5 of [2] in the case when $\Gamma=\Lambda$, except that a side condition was assumed to be adequate, and not strongly adequate. It was shown that this forcing poset preserves $\omega_{1}$ and $\omega_{2}$ and adds a total function from $\omega_{2}$ to $\omega_{2}$. The proof relied on the fact that remainder sets are subsets of $\Gamma$ 
when $\Lambda=\Gamma$. Definition 3.4 gives a version of this forcing poset in the case when $\Gamma$ is a proper subset of $\Lambda$, but we require a side condition to be strongly adequate. Under this stronger requirement, the proof of the preservation of cardinals is identical to that in [2].

\section{Adding a Club to a fat stationary set}

Fix for the remainder of the section a fat stationary set $S \subseteq \omega_{2}$. Being fat means that for every club $D \subseteq \omega_{2}, S \cap D$ contains a closed subset of order type $\omega_{1}+1$. We will define a forcing poset with finite conditions which adds a club subset of $S$. Clearly it suffices to add a club to any stationary subset of $S$. By fatness and the fact that $\Lambda$ contains almost all points of cofinality $\omega_{1}$, there are stationarily many $\beta$ in $S \cap \operatorname{cof}\left(\omega_{1}\right)$ such that $\beta \in \Lambda$ and $S \cap \operatorname{cof}(\omega) \cap \beta$ contains a club in $\beta$. By removing the ordinals where this fails, we will assume without loss of generality that this property holds for all $\beta$ in $S \cap \operatorname{cof}\left(\omega_{1}\right)$.

Let $\Gamma=S \cap \operatorname{cof}\left(\omega_{1}\right)$. Then $\Gamma$ is a stationary subset of $\Lambda$. Let us define the comparison points $\beta_{M, N}$ for $M$ and $N$ in $\mathcal{X}$ relative to this set $\Gamma$. Let $\mathcal{Z}$ denote the set of $M$ in $\mathcal{X}$ such that $\sup (M) \in S$ and for all $\beta \in M \cap S, \sup (M \cap \beta) \in S$. Note that if $M \in \mathcal{Z}$ and $\beta \in M \cap \Gamma$, then $M \cap \beta \in \mathcal{Z}$.

Lemma 4.1. The set $\mathcal{Z}$ is stationary in $P_{\omega_{1}}\left(\omega_{2}\right)$.

Proof. Let $F:\left[\omega_{2}\right]^{<\omega} \rightarrow \omega_{2}$ be a function. Since $\Gamma$ is stationary, we can find $\beta \in \Gamma$ which is closed under $F$. As $\mathcal{X}$ is stationary, fix $N^{*}$ which is countable and satisfies that $N^{*} \prec\left(H\left(\omega_{2}\right), \in, F, S\right), \beta \in N^{*}$, and $N:=N^{*} \cap \omega_{2} \in \mathcal{X}$.

By elementarity, there is a club subset $c$ of $\beta$ in $N^{*}$ such that $c \subseteq S$. Again by elementarity, $N \cap c$ is unbounded in $\sup (N \cap \beta)$, so $\sup (N \cap \beta)$ is in $c$ and hence in $S$. Let $M=N \cap \beta$. Then $M$ is in $\mathcal{X}$ and $\sup (M)=\sup (N \cap \beta) \in S$. Also since $N$ and $\beta$ are both closed under $F$, so is $M$.

Let $\gamma \in M \cap S$ be given. If $\gamma$ has cofinality $\omega$, then $\sup (M \cap \gamma)=\gamma$, which is in $S$. Assume $\gamma$ has cofinality $\omega_{1}$. By elementarity, there is a club subset $d$ of $\gamma$ in $N^{*}$ such that $d \subseteq S$. Then $N \cap d$ is unbounded in $\sup (N \cap \gamma)$, so $\sup (N \cap \gamma)$ is in $d$ and hence in $S$. Since $\gamma<\beta, \sup (N \cap \gamma)=\sup (M \cap \gamma)$.

Given ordinals $\alpha \leq \gamma$ and $\alpha^{\prime} \leq \gamma^{\prime}$, we say that the pairs $\langle\alpha, \gamma\rangle$ and $\left\langle\alpha^{\prime}, \gamma^{\prime}\right\rangle$ are overlapping if $[\alpha, \gamma] \cap\left[\alpha^{\prime}, \gamma^{\prime}\right] \neq \emptyset$, and are nonoverlapping if $[\alpha, \gamma] \cap\left[\alpha^{\prime}, \gamma^{\prime}\right]=\emptyset$.

Definition 4.2. Let $\mathbb{P}$ be the forcing poset whose conditions are pairs $(x, A)$ satisfying:

(1) $x$ is a finite set of nonoverlapping pairs $\langle\alpha, \gamma\rangle$, where $\alpha \in S$ and $\alpha \leq \gamma<\omega_{2}$;

(2) $A$ is a finite strongly adequate subset of $\mathcal{Z}$;

(3) if $M \in A,\langle\alpha, \gamma\rangle \in x$, and $M \cap[\alpha, \gamma] \neq \emptyset$, then $\alpha$ and $\gamma$ are in $M$;

(4) if $M \in A,\langle\alpha, \gamma\rangle \in x, M \cap[\alpha, \gamma]=\emptyset$, and $M \backslash \alpha$ is nonempty, then $\min (M \backslash \alpha) \in S$.

Let $(y, B) \leq(x, A)$ if $x \subseteq y$ and $A \subseteq B$.

Note that in (4), the ordinal $\min (M \backslash \alpha)$ has cofinality $\omega_{1}$. So the conclusion of

(4) is equivalent to requiring that $\min (M \backslash \alpha)$ is in $\Gamma$.

If $p=(x, A)$, we let $x_{p}=x$ and $A_{p}=A$.

Let $\dot{D}$ be a $\mathbb{P}$-name such that $\mathbb{P}$ forces

$$
\dot{D}=\left\{\alpha: \exists p \in \dot{G} \exists \gamma\langle\alpha, \gamma\rangle \in x_{p}\right\}
$$


Our goal is to show that $\mathbb{P}$ preserves cardinals and forces that $\dot{D}$ is a club subset of $S$.

Lemma 4.3. Let $p$ be a condition. Suppose that $\langle\alpha, \gamma\rangle \in x_{p}, N \in A_{p}, N \cap[\alpha, \gamma]=\emptyset$, and $N \backslash \alpha \neq \emptyset$. Let $\beta=\min (N \backslash \alpha)$. Then either $\langle\beta, \xi\rangle \in x_{p}$ for some $\xi$, or $\left(x_{p} \cup\{\langle\beta, \beta\rangle\}, A_{p}\right)$ is a condition below $p$.

Proof. Suppose that there is no pair of the form $\langle\beta, \xi\rangle$ in $x_{p}$, and we will prove that $\left(x_{p} \cup\{\langle\beta, \beta\rangle\}, A_{p}\right)$ is a condition. (1) Since $p$ is a condition, $\beta \in \Gamma$. Consider a pair $\langle\xi, \zeta\rangle \in x_{p}$, and suppose for a contradiction that $\beta \in[\xi, \zeta]$. Then $N \cap[\xi, \zeta] \neq \emptyset$ and $\xi<\beta \leq \zeta$. It follows that $\xi \in N \cap \beta$. Since $\beta=\min (N \backslash \alpha)$ and $\xi<\beta$, we must have that $\xi<\alpha$. But then $\xi<\alpha<\beta \leq \zeta$. Hence $\langle\alpha, \gamma\rangle$ and $\langle\xi, \zeta\rangle$ are distinct and overlapping, which contradicts that $p$ is a condition.

(2) and (3) are immediate. For (4), suppose that $M \in A_{p}, \beta \notin M$, and $M \backslash \beta$ is nonempty. We need to show that $\min (M \backslash \beta) \in \Gamma$. Since $\beta \in N$, if $\beta \geq \beta_{M, N}$ then $\min (M \backslash \beta)$ is in $R_{N}(M)$ and hence in $\Gamma$. Suppose that $\beta<\beta_{M, N}$. Then since $\beta \in N \backslash M, M \cap \beta_{M, N} \in S k(N)$. So $M \cap \beta_{M, N} \subseteq N$. As $\gamma<\beta<\beta_{M, N}$ and $N \cap[\alpha, \gamma]=\emptyset$, it follows that $M \cap[\alpha, \gamma]=\emptyset$. Since $p$ is a condition, $\min (M \backslash \alpha) \in \Gamma$. But as $M \cap \beta \subseteq N \cap \beta$ and $\beta=\min (N \backslash \alpha), \min (M \backslash \alpha)=\min (M \backslash \beta)$.

Proposition 4.4. The forcing poset $\mathbb{P}$ preserves $\omega_{1}$.

Proof. Let $p \Vdash \dot{g}: \omega \rightarrow \omega_{1}$ is a function. Fix $\theta>\omega_{2}$ regular with $\dot{g} \in H(\theta)$. Let $N^{*}$ be a countable elementary substructure of $H(\theta)$ satisfying that $\mathbb{P}, p, \dot{g}, \pi, \mathcal{X}, S, \mathcal{Z} \in$ $N^{*}$ and $N:=N^{*} \cap \omega_{2} \in \mathcal{Z}$. Note that since $\pi \in N^{*}, N^{*} \cap H\left(\omega_{2}\right)=\pi\left[N \cap \omega_{2}\right]=$ $S k(N)$. In particular, $N^{*} \cap \mathbb{P} \subseteq S k(N)$.

Define $q=\left(x_{p}, A_{p} \cup\{N\}\right)$. We will prove that $q$ is $N^{*}$-generic. Then $q$ forces that the range of $\dot{g}$ is contained in $N$, so $\dot{g}$ does not collapse $\omega_{1}$. Fix a dense set $D \in N^{*}$, and we will show that $N^{*} \cap D$ is predense below $q$.

Let $r \leq q$. Applying Lemma 4.3 finitely many times, we will assume without loss of generality that whenever $\langle\alpha, \gamma\rangle \in x_{r}, N \in A_{r}, N \cap[\alpha, \gamma]=\emptyset$, and $N \backslash \alpha$ is nonempty, then $\langle\min (N \backslash \alpha), \xi\rangle$ is in $x_{r}$ for some $\xi$.

Define $s=\left(x_{r}, A^{\prime}\right)$, where

$$
A^{\prime}=A_{r} \cup\left\{M \cap \beta_{M, N}: M \in A_{r}, M \cap \beta_{M, N} \in S k(N)\right\} .
$$

We claim that $s$ is a condition. (1) is immediate. (2) $A^{\prime}$ is strongly adequate by Proposition 2.5. Let us prove that $A^{\prime} \subseteq \mathcal{Z}$. Consider $M \in A_{r}$ such that $M \cap \beta_{M, N} \in S k(N)$. First we show that $\sup \left(M \cap \beta_{M, N}\right) \in S$. If $M=M \cap \beta_{M, N}$ then this is immediate. Otherwise let $\beta=\min \left(M \backslash \beta_{M, N}\right)$. Then $\beta \in R_{N}(M)$, and hence $\beta \in \Gamma$ since $A$ is strongly adequate. As $M \in \mathcal{Z}$ and $\beta \in M \cap \Gamma$, $\sup \left(M \cap \beta_{M, N}\right)=\sup (M \cap \beta)$ is in $S$. Now if $\gamma \in\left(M \cap \beta_{M, N}\right) \cap S$, then $\gamma \in M \cap S$ and therefore $\sup \left(\left(M \cap \beta_{M, N}\right) \cap \gamma\right)=\sup (M \cap \gamma) \in S$.

$(3,4)$ Consider $M \in A_{r}$ such that $M \cap \beta_{M, N} \in S k(N)$, and let $\langle\alpha, \gamma\rangle \in x_{r}$. First suppose that $\left(M \cap \beta_{M, N}\right) \cap[\alpha, \gamma] \neq \emptyset$. Then $\alpha$ and $\gamma$ are in $M$. Since $M \cap \beta_{M, N} \in S k(N), N \cap[\alpha, \gamma] \neq \emptyset$. So $\alpha$ and $\gamma$ are also in $N$. Hence $\alpha$ and $\gamma$ are in $M \cap N$, which is a subset of $\beta_{M, N}$. So $\alpha$ and $\gamma$ are in $M \cap \beta_{M, N}$. Now suppose that $\left(M \cap \beta_{M, N}\right) \cap[\alpha, \gamma]=\emptyset$. If $M \cap[\alpha, \gamma] \neq \emptyset$, then $\alpha$ is in $M \backslash\left(M \cap \beta_{M, N}\right)$, so $\alpha \geq \beta_{M, N}$. In that case, $\min \left(\left(M \cap \beta_{M, N}\right) \backslash \alpha\right)$ does not exist and we are done. On the other hand, if $M \cap[\alpha, \gamma]=\emptyset$ and $\min \left(\left(M \cap \beta_{M, N}\right) \backslash \alpha\right)$ exists, then clearly $\min \left(\left(M \cap \beta_{M, N}\right) \backslash \alpha\right)$ is equal to $\min (M \backslash \alpha)$, which is in $S$. This completes the proof that $s$ is a condition. 
Let $u=\left(x_{s} \cap S k(N), A_{s} \cap S k(N)\right)$. Then $u \in N^{*} \cap \mathbb{P}$. Let $R(N)=\bigcup\left\{R_{M}(N)\right.$ : $\left.M \in A_{s}\right\}$. Then $R(N)$ is a finite subset of $N$ and hence is in $N^{*}$. Since $\mathcal{Z} \in N^{*}$, by elementarity we can fix $K \in N^{*} \cap \mathcal{Z}$ satisfying that $u \in S k(K)$ and $R(N) \subseteq K$. Since $R(N) \subseteq K \cap \Gamma$, for all $\zeta \in R(N), K \cap \zeta \in \mathcal{Z}$. Define $v=\left(x_{u}, A^{*}\right)$, where

$$
A^{*}=A_{u} \cup\{K\} \cup\{K \cap \zeta: \zeta \in R(N)\} .
$$

Let us prove that $v$ is a condition. (1) is clear and (2) follows from Proposition 2.10. $(3,4)$ Let $\langle\alpha, \gamma\rangle$ be in $x_{u}$. Then $\alpha, \gamma \in K$. Fix $\zeta \in R(N)$, and suppose that $(K \cap \zeta) \cap[\alpha, \gamma]=\emptyset$. Since $\alpha \in K, \alpha \geq \zeta$. So $\min ((K \cap \zeta) \backslash \alpha)$ does not exist. This proves (4).

For (3), fix $\zeta \in R_{M}(N)$ for some $M \in A_{s}$, and suppose that $(K \cap \zeta) \cap[\alpha, \gamma] \neq \emptyset$. Then $\alpha<\zeta$. We need to show that $\alpha$ and $\gamma$ are in $K \cap \zeta$. Since $\alpha$ and $\gamma$ are in $K$, it suffices to show that $\gamma<\zeta$. Recall that by the definition of $R_{M}(N), \beta_{M, N} \leq \zeta$.

Suppose for a contradiction that $\zeta \leq \gamma$. So we have that $\alpha<\zeta \leq \gamma$. In particular, $\beta_{M, N} \leq \gamma$. We claim that $M \cap[\alpha, \gamma]=\emptyset$. If not, then $\gamma \in M$ since $s$ is a condition; but this is impossible since then $\gamma \in M \cap N \subseteq \beta_{M, N} \leq \zeta$. So indeed $M \cap[\alpha, \gamma]=\emptyset$.

Suppose that $\beta_{M, N} \leq \alpha$. Then since $\alpha<\zeta$ are in $N$, it cannot be the case that $\zeta=\min \left(N \backslash \beta_{M, N}\right)$. So there is $\sigma \geq \beta_{M, N}$ in $M$ such that $\zeta=\min (N \backslash \sigma)$. Since $\alpha \in N$ and $\alpha<\zeta$, this implies that $\alpha<\sigma<\zeta$. But then $\sigma \in M \cap[\alpha, \gamma]$, contradicting that $M \cap[\alpha, \gamma]=\emptyset$.

Therefore we have that $\alpha<\beta_{M, N} \leq \zeta$. So $\alpha$ is in $N \cap \beta_{M, N}$ but is not in $M$. This implies that $M \cap \beta_{M, N} \in S k(N)$. The definition of $R_{M}(N)$ in this case gives that there is $\sigma \in M \backslash \beta_{M, N}$ such that $\zeta=\min (N \backslash \sigma)$. But then we have that $\alpha<\beta_{M, N} \leq \sigma<\zeta \leq \gamma$; so $M \cap[\alpha, \gamma]$ is nonempty, which again is a contradiction. Thus $\gamma<\zeta$. So $\gamma \in K \cap \zeta$, which completes the proof that $v$ is a condition.

Fix $w \leq v$ in $N^{*} \cap D$. We will prove that $w$ and $s$ are compatible, which shows that $N^{*} \cap D$ is predense below $q$. So let $z=\left(x_{z}, A_{z}\right)$ be defined by letting $x_{z}=x_{w} \cup x_{s}$ and $A_{z}=A_{w} \cup A_{s}$. We will prove that $z$ is a condition. Then clearly $z \leq w, s$ and we are done.

(1) Let $\langle\alpha, \gamma\rangle \in x_{w}$ and $\left\langle\alpha^{\prime}, \gamma^{\prime}\right\rangle \in x_{s}$ be given. Suppose for a contradiction that the pairs are distinct and overlapping. If $N \cap\left[\alpha^{\prime}, \gamma^{\prime}\right] \neq \emptyset$, then $\alpha^{\prime}$ and $\gamma^{\prime}$ are in $N$. It follows that $\left\langle\alpha^{\prime}, \gamma^{\prime}\right\rangle \in x_{w}$ and the intervals are nonoverlapping since $w$ is a condition, which is a contradiction. So $N \cap\left[\alpha^{\prime}, \gamma^{\prime}\right]=\emptyset$. Since $\alpha$ and $\gamma$ are in $N$, we must have that $\alpha<\alpha^{\prime} \leq \gamma^{\prime}<\gamma$. Let $\beta=\min \left(N \backslash \alpha^{\prime}\right)$. Then $\alpha<\gamma^{\prime}<\beta \leq \gamma$, and by the choice of $r,\langle\beta, \xi\rangle \in x_{s}$ for some $\xi$. Since $\beta \in N,\langle\beta, \xi\rangle \in x_{w}$. But then $\langle\alpha, \gamma\rangle$ and $\langle\beta, \xi\rangle$ are distinct pairs in $x_{w}$ which overlap, contradicting that $w$ is a condition.

(2) $A_{z}$ is strongly adequate by Proposition 2.11 , and clearly $A_{z} \subseteq \mathcal{Z}$. $(3,4)$ Let $M \in A_{w}$ and $\langle\alpha, \gamma\rangle \in x_{s}$. If $N \cap[\alpha, \gamma] \neq \emptyset$ then $\alpha$ and $\gamma$ are in $N$, and so $\langle\alpha, \gamma\rangle \in x_{w}$. In this case (3) and (4) are satisfied since $w$ is a condition.

Suppose that $N \cap[\alpha, \gamma]=\emptyset$. Then since $M \in S k(N), M \cap[\alpha, \gamma]=\emptyset$. Assume that $\beta:=\min (M \backslash \alpha)$ exists, and we will prove that $\beta \in \Gamma$. Let $\beta^{\prime}=\min (N \backslash \alpha)$. Then $\beta^{\prime} \in \Gamma$. If $\beta=\beta^{\prime}$ then we are done. Suppose $\beta^{\prime}<\beta$. By the choice of $r$, $\left\langle\beta^{\prime}, \xi\right\rangle \in x_{s}$ for some $\xi$, and as $\beta^{\prime} \in N,\left\langle\beta^{\prime}, \xi\right\rangle \in x_{w}$. Since $\beta^{\prime} \notin M$, the ordinal $\min \left(M \backslash \beta^{\prime}\right)$, which is equal to $\beta$, is in $\Gamma$.

Now let $M \in A_{s}$ and $\langle\alpha, \gamma\rangle \in x_{w}$. Suppose that $M \cap[\alpha, \gamma] \neq \emptyset$, and we show that $\alpha$ and $\gamma$ are in $M$. 
First assume that there is $\xi \in M \cap[\alpha, \gamma]$ such that $\xi \geq \beta_{M, N}$. Let $\zeta=\min (N \backslash$ $\xi)$. Then $\zeta \in R_{M}(N)$ and $\zeta \in(\alpha, \gamma]$. By the choice of $K, \zeta \in K$. Therefore $K \cap[\alpha, \gamma] \neq \emptyset$. Since $K \in A_{w}, \alpha$ and $\gamma$ are in $K$. But $\alpha<\zeta$, so $\alpha \in K \cap \zeta$. Hence $(K \cap \zeta) \cap[\alpha, \gamma] \neq \emptyset$. Since $K \cap \zeta \in A_{w}, \gamma \in K \cap \zeta$. But this is impossible since $\zeta \leq \gamma$. So this configuration is not possible.

Therefore $M \cap[\alpha, \gamma] \subseteq \beta_{M, N}$. Since $M \cap[\alpha, \gamma]$ is nonempty, $\left(M \cap \beta_{M, N}\right)$ meets the interval $[\alpha, \gamma]$. If $M \cap \beta_{M, N} \in S k(N)$, then $M \cap \beta_{M, N}$ is in $A_{w}$, and therefore $\alpha$ and $\gamma$ are in $M \cap \beta_{M, N}$ and hence in $M$. Otherwise $N \cap \beta_{M, N}$ is either equal to $M \cap \beta_{M, N}$ or is in $S k(M)$. If $\beta_{M, N}>\gamma$, this implies that $\alpha$ and $\gamma$ are in $N \cap \beta_{M, N}$ and hence in $M$.

Otherwise we have that $\alpha<\beta_{M, N} \leq \gamma$. Let $\zeta=\min \left(N \backslash \beta_{N, M}\right)$. Then $\zeta \in$ $R_{M}(N)$ and $\alpha<\zeta \leq \gamma$. By the choice of $K, \zeta \in K$, and hence $K$ meets the interval $[\alpha, \gamma]$. Therefore $\alpha \in K$ since $w$ is a condition. So $\alpha \in K \cap \zeta$, and therefore $K \cap \zeta$ meets the interval $[\alpha, \gamma]$. This implies that $\gamma \in K \cap \zeta$ since $w$ is a condition, contradicting that $\zeta \leq \gamma$.

Now assume that $M \cap[\alpha, \gamma]=\emptyset$ and $\min (M \backslash \alpha)$ exists. We will prove that $\min (M \backslash \alpha) \in \Gamma$. Assume first that $N \cap \beta_{M, N}$ is either equal to $M \cap \beta_{M, N}$ or is in $S k(M)$. Since $\alpha$ is in $N \backslash M, \beta_{M, N} \leq \alpha$. So $\alpha \in N \backslash \beta_{M, N}$, and thus $\min (M \backslash \alpha)$ is in $R_{N}(M)$ and hence in $\Gamma$. Now suppose that $M \cap \beta_{M, N} \in S k(N)$. Then $M \cap \beta_{M, N} \in A_{w}$. If $\beta_{M, N} \leq \alpha$, then again $\min (M \backslash \alpha)$ is in $R_{N}(M)$ and hence in $\Gamma$. So assume that $\alpha<\beta_{M, N}$. If $\min \left(\left(M \cap \beta_{M, N}\right) \backslash \alpha\right)$ exists, then clearly it is equal to $\min (M \backslash \alpha)$, and this ordinal is in $\Gamma$ since $w$ is a condition. Otherwise the least element of $M$ above $\alpha$ is at least $\beta_{M, N}$. But then $\min (M \backslash \alpha)=\min \left(M \backslash \beta_{M, N}\right)$, which is in $R_{N}(M)$ and hence in $\Gamma$.

Proposition 4.5. The forcing poset $\mathbb{P}$ preserves $\omega_{2}$.

Proof. Let $p \Vdash \dot{g}: \omega_{1} \rightarrow \omega_{2}$ is a function. Fix $\theta>\omega_{2}$ regular such that $\dot{g} \in H(\theta)$. Let $N^{*} \prec H(\theta)$ be of size $\omega_{1}$ such that $\mathbb{P}, p, \dot{g}, \pi, \mathcal{X}, S, \mathcal{Z} \in N^{*}$ and $\beta^{*}:=N^{*} \cap \omega_{2} \in$ $\Gamma$. This is possible since $\Gamma$ is stationary. Note that since $\pi \in N^{*}, S k\left(\beta^{*}\right)=\pi\left[\beta^{*}\right]=$ $N^{*} \cap H\left(\omega_{2}\right)$. In particular, $N^{*} \cap \mathbb{P} \subseteq S k\left(\beta^{*}\right)$.

Fix $K \in \mathcal{Z}$ such that $p$ and $\beta^{*}$ are in $S k(K)$. Let $q=\left(x_{q}, A_{q}\right)$, where $x_{q}=$ $x_{p} \cup\left\{\left\langle\beta^{*}, \beta^{*}\right\rangle\right\}$ and $A_{q}=A_{p} \cup\{K\} \cup\{K \cap \beta\}$. Then $A_{q}$ is strongly adequate by Lemma 2.12. The other properties of being a condition are easy to check. We claim that $q$ is $N^{*}$-generic. This implies that $q$ forces that the range of $\dot{g}$ is a subset of $N^{*}$, and hence does not collapse $\omega_{2}$. Fix a dense set $D \in N^{*}$, and we will show that $N^{*} \cap D$ is predense below $q$.

Let $r \leq q$ be given, and we will find a condition $w$ in $N^{*} \cap D$ which is compatible with $r$. Note that for any pair $\langle\alpha, \gamma\rangle$ in $x_{r}$ different from $\left\langle\beta^{*}, \beta^{*}\right\rangle$, $\alpha$ and $\gamma$ are either both below or both above $\beta^{*}$.

Let $s=\left(x_{r}, A^{*}\right)$, where

$$
A^{*}=\left\{M \cap \beta^{*}: M \in A_{r}\right\} .
$$

We claim that $s$ is a condition. (1) is immediate. For (2), the set $A_{s}$ is strongly adequate by Proposition 2.14. To see that $A_{s} \subseteq \mathcal{Z}$, consider $M \in A_{r}$. If $M=$ $M \cap \beta^{*}$ then we are done. Otherwise let $\xi=\min \left(M \backslash \beta^{*}\right)$. Then $\xi$ is in $\Gamma$; for if $\xi=\beta^{*}$ then this is immediate, and otherwise $\xi \in \Gamma$ by property (4) of $r$ being a condition. Now $M \cap \beta^{*}=M \cap \xi$, so $\sup \left(M \cap \beta^{*}\right) \in S$ since $M \in \mathcal{Z}$. Suppose $\zeta$ is in $\left(M \cap \beta^{*}\right) \cap \Gamma$. Then $\zeta \in M \cap \Gamma$. But $\sup \left(\left(M \cap \beta^{*}\right) \cap \zeta\right)=\sup (M \cap \zeta)$, which is in $S$ since $M \in \mathcal{Z}$. 
$(3,4)$ Consider $\langle\alpha, \gamma\rangle \in x_{r}$ and $M \in A_{r}$. If $\alpha$ and $\gamma$ are at least $\beta^{*}$, then $\left(M \cap \beta^{*}\right) \cap[\alpha, \gamma]=\emptyset$ and the least element of $M \cap \beta^{*}$ above $\alpha$ does not exist. Suppose that $\alpha$ and $\gamma$ are both below $\beta^{*}$. If $\left(M \cap \beta^{*}\right) \cap[\alpha, \gamma]=\emptyset$ and $\min \left(\left(M \cap \beta^{*}\right) \backslash \alpha\right)$ exists, then $M \cap[\alpha, \gamma]=\emptyset$ and $\min \left(\left(M \cap \beta^{*}\right) \backslash \alpha\right)=\min (M \backslash \alpha) \in \Gamma$. If $\left(M \cap \beta^{*}\right) \cap[\alpha, \gamma] \neq \emptyset$, then $\alpha$ and $\gamma$ are in $M$, and hence in $M \cap \beta^{*}$. This completes the proof that $s$ is a condition.

Let $v=\left(x_{s} \cap S k\left(\beta^{*}\right), A_{s} \cap S k\left(\beta^{*}\right)\right)$. So $x_{v}=x_{s} \cap\left(\beta^{*} \times \beta^{*}\right)$ and $A_{v}=A_{s} \cap P\left(\beta^{*}\right)$. By elementarity, fix $w \leq v$ in $N^{*} \cap D$. Let $z=\left(x_{w} \cup x_{s}, A_{w} \cup A_{s}\right)$. We will prove that $z$ is a condition. Then clearly $z \leq w, s$, completing the proof.

(1) Let $\langle\alpha, \gamma\rangle \in x_{w}$ and $\left\langle\alpha^{\prime}, \gamma^{\prime}\right\rangle \in x_{s}$ be distinct. If $\alpha^{\prime}$ and $\gamma^{\prime}$ are both at least $\beta^{*}$, then obviously $[\alpha, \gamma] \cap\left[\alpha^{\prime}, \gamma^{\prime}\right]=\emptyset$. Otherwise they are both below $\beta^{*}$, and so $\left\langle\alpha^{\prime}, \gamma^{\prime}\right\rangle \in x_{w}$. Then $[\alpha, \gamma] \cap\left[\alpha^{\prime}, \gamma^{\prime}\right]=\emptyset$ since $w$ is a condition. (2) $A_{z}$ is strongly adequate by Proposition 2.15, and clearly $A_{z} \subseteq \mathcal{Z}$.

$(3,4)$ Let $M \in A_{w}$ and $\langle\alpha, \gamma\rangle \in x_{s}$. If $\alpha$ and $\gamma$ are at least $\beta^{*}$, then $M \cap[\alpha, \gamma]=\emptyset$ and $\min (M \backslash \alpha)$ does not exist. Suppose $\alpha$ and $\gamma$ are both below $\beta^{*}$. Then $\langle\alpha, \gamma\rangle \in$ $x_{w}$, so (3) and (4) are satisfied for $\langle\alpha, \gamma\rangle$ and $M$ since $w$ is a condition.

Now let $M \in A_{s}$ and $\langle\alpha, \gamma\rangle \in x_{w}$. Then $\alpha$ and $\gamma$ are below $\beta^{*}$ and $M \cap \beta^{*} \in A_{w}$. Suppose that $M \cap[\alpha, \gamma]=\emptyset$. Then $\left(M \cap \beta^{*}\right) \cap[\alpha, \gamma]=\emptyset$. If $\min (M \backslash \alpha)$ does not exist then we are done. If $\min \left(\left(M \cap \beta^{*}\right) \backslash \alpha\right)$ exists, then it is in $\Gamma$ and it is equal to $\min (M \backslash \alpha)$. Otherwise $\min (M \backslash \alpha)$ exists but $\min \left(\left(M \cap \beta^{*}\right) \backslash \alpha\right)$ does not exist. So $\min (M \backslash \alpha) \geq \beta^{*}$, and therefore $\min (M \backslash \alpha)=\min \left(M \backslash \beta^{*}\right)$. If $\beta^{*} \in M$, then $\min (M \backslash \alpha)=\beta^{*}$, which is in $\Gamma$. Otherwise $\min (M \backslash \alpha)=\min \left(M \backslash \beta^{*}\right)$ is in $\Gamma$ since $s$ is a condition and $\left\langle\beta^{*}, \beta^{*}\right\rangle$ is in $x_{s}$. Now suppose that $M \cap[\alpha, \gamma] \neq \emptyset$. Since $\alpha$ and $\gamma$ are below $\beta^{*},\left(M \cap \beta^{*}\right) \cap[\alpha, \gamma] \neq \emptyset$. Since $M \cap \beta^{*} \in A_{w}, \alpha$ and $\gamma$ are in $M \cap \beta^{*}$ and hence in $M$.

Finally, we show that $\mathbb{P}$ adds a club subset of $S$. Recall that $\dot{D}$ is a $\mathbb{P}$-name and $\mathbb{P}$ forces that

$$
\dot{D}=\left\{\alpha: \exists p \in \dot{G} \exists \gamma\langle\alpha, \gamma\rangle \in x_{p}\right\}
$$

Clearly $\dot{D}$ is forced to be a subset of $S$, and it is straightforward to verify that $\dot{D}$ is forced to be cofinal in $\omega_{2}$. Proposition 4.7 below will show that $\dot{D}$ is a club.

Lemma 4.6. Suppose that $p$ forces that $\alpha$ is a limit point of $\dot{D}$. Assume that $M \in A_{p}$ and $\sup (M \cap \alpha)=\alpha$. Then $\sup (M \cap S \cap \alpha)=\alpha$.

Proof. Let $\xi<\alpha$ be given, and we will find an element of $M \cap S \cap \alpha$ which is above $\xi$. Since $p$ forces that $\alpha$ is a limit point of $\dot{D}$, we can find $q \leq p$, and $\sigma$ and $\pi$ such that $\xi<\sigma \leq \pi<\alpha$ and $\langle\sigma, \pi\rangle \in x_{q}$. If $\sigma \in M$ then we are done, since $\sigma \in S$. Otherwise $M \cap[\sigma, \pi]=\emptyset$ and $\min (M \backslash \sigma)$ is in $S$. Since $\xi<\sigma$ and $M \cap \alpha$ is cofinal in $\alpha$, we have that $\xi<\min (M \backslash \sigma)<\alpha$.

Proposition 4.7. The forcing poset $\mathbb{P}$ forces that $\dot{D}$ is closed.

Proof. It will suffice to show that if $p$ forces that $\alpha$ is a limit point of $\dot{D}$, then there is $q \leq p$ such that $\langle\alpha, \gamma\rangle \in x_{q}$ for some $\gamma$. If $p$ already satisfies this property, then we are done; so assume not. Then since $p$ forces that $\alpha$ is a limit point of $\dot{D}$, for any pair $\langle\xi, \gamma\rangle \in x_{p}$, the ordinals $\xi$ and $\gamma$ are either both below or both strictly above $\alpha$. In particular, the pair $\langle\alpha, \alpha\rangle$ does not overlap any pair in $x_{p}$. Also note that $\alpha$ is a limit point of $S$, since this is true in a generic extension.

We claim that for any $M \in A_{p}$, if $\sup (M \cap \alpha)<\alpha$ and $M \backslash \alpha \neq \emptyset$, then $\min (M \backslash \alpha) \in \Gamma$. Namely, fix $r \leq p$ and $\langle\xi, \gamma\rangle \in x_{r}$ such that $\sup (M \cap \alpha)<\xi \leq \gamma<$ 
$\alpha$. Then since $M \in A_{r}$ and $M \cap[\xi, \gamma]=\emptyset, \min (M \backslash \xi) \in \Gamma$. But as $\sup (M \cap \alpha)<\xi$, $\min (M \backslash \xi)=\min (M \backslash \alpha)$.

First suppose that there exists $M \in A_{p}$ such that $\sup (M)=\alpha$. Then since $M \in \mathcal{Z}, \sup (M)=\alpha \in S$. Let $q=\left(x_{p} \cup\{\langle\alpha, \alpha\rangle\}, A_{p}\right)$. We will show that $q$ is a condition, which finishes the proof in this case.

The only way that $q$ could fail to be a condition is if there is some $N \in A_{p}$ such that $\alpha \notin N$ and $\xi:=\min (N \backslash \alpha) \notin \Gamma$. We will show that this is impossible. By the comments above, we must have that $\sup (N \cap \alpha)=\alpha$. So $\alpha$ is a common limit point of $M$ and $N$, which implies that $\alpha<\beta_{M, N}$. First assume that $\xi<\beta_{M, N}$. Then since $\xi \in N \backslash M, M \cap \beta_{M, N}=M \in S k(N)$. By elementarity, this implies that $\sup (M)=\alpha$ is in $N$, which contradicts our assumptions. Now assume that $\beta_{M, N} \leq \xi$. Then both $M \cap \beta_{M, N}$ and $N \cap \beta_{M, N}$ have a supremum equal to $\alpha$, which belongs to neither $M$ nor $N$. Hence the only comparison of $M$ and $N$ which is possible is that $M \cap \beta_{M, N}=N \cap \beta_{M, N}$. But then $\xi=\min \left(N \backslash \beta_{M, N}\right)$ is in $R_{M}(N)$ and hence in $\Gamma$, which again contradicts our assumptions.

We now assume that for all $M \in A_{p}$, if $\sup (M \cap \alpha)=\alpha$, then $M \backslash \alpha$ is nonempty. Recall that if $M \in A_{p}, \sup (M \cap \alpha)<\alpha$, and $M \backslash \alpha$ is nonempty, then $\min (M \backslash \alpha) \in \Gamma$. Define $A_{0}, A_{1}$, and $A_{2}$ as follows:

$$
\begin{gathered}
A_{0}=\left\{M \in A_{p}: \sup (M)<\alpha\right\}, \\
A_{1}=\left\{M \in A_{p}: \sup (M \cap \alpha)<\alpha, \min (M \backslash \alpha) \in \Gamma\right\}, \\
A_{2}=\left\{M \in A_{p}: \sup (M \cap \alpha)=\alpha, M \backslash \alpha \neq \emptyset\right\} .
\end{gathered}
$$

Then by the facts just noted, $A_{p}=A_{0} \cup A_{1} \cup A_{2}$.

First suppose that $A_{2}$ is empty. We claim that $q=\left\langle x_{p} \cup\{\langle\alpha, \alpha\rangle\}, A_{p}\right\rangle$ is a condition, which will finish the proof in this case. Clearly $q$ is a condition unless $\alpha$ is not in $S$, since the other properties are immediate. Suppose that $\alpha$ is not in $S$. Then for all $M \in A_{p}$, either $\sup (M)<\alpha$ in case $M \in A_{0}$, or $\sup (M \cap \alpha)<\alpha$ and $\min (M \backslash \alpha) \in \Gamma$ in case $M \in A_{1}$. In the second case, $\min (M \backslash \alpha)>\alpha$ since $\alpha$ is not in $S$. Fix $\xi<\alpha$ in $S$ larger than $\sup (M \cap \alpha)$ for all $M \in A_{p}$. Then easily $\left(x_{p} \cup\{\langle\xi, \alpha\rangle\}, A_{p}\right)$ is a condition, contradicting that $p$ forces that $\alpha$ is a limit point of $\dot{D}$.

Now suppose that $A_{2}$ is nonempty. Let $q=\left(x_{p} \cup\{\langle\alpha, \alpha\rangle\}, A_{p}\right)$. If $q$ is a condition then we are done, so assume that it is not. Then either $\alpha$ is not in $S$, or there is $N \in A_{p}$ such that $\alpha \notin N$ and $\min (N \backslash \alpha)$ is not in $S$. The rest of the proof will lead us to a contradiction.

Let $M$ be $\in$-minimal in $A_{2}$ and let $\xi:=\min (M \backslash \alpha)$. We claim that $\xi$ is not in $S$. So suppose that it is. Then since $M \in \mathcal{Z}, \sup (M \cap \xi)=\alpha \in S$. Therefore there must be $N \in A_{p}$ with $\alpha \notin N$ and $\min (N \backslash \alpha) \notin S$. Note that $N$ must be in $A_{2}$. So $\alpha$ is a common limit point of $M$ and $N$, which implies that $\alpha<\beta_{M, N}$. By the $\in$ minimality of $M$, either $M \cap \beta_{M, N}=N \cap \beta_{M, N}$ or $M \cap \beta_{M, N} \in S k(N)$. But $M \cap \beta_{M, N}$ cannot be in $S k(N)$, since if it were then $M \cap \alpha$, and hence $\sup (M \cap \alpha)=\alpha$, would be in $N$. So $M \cap \beta_{M, N}=N \cap \beta_{M, N}$. Since $\xi=\min (M \backslash \alpha) \in S$ and $\min (N \backslash \alpha) \notin S$, it must be the case that $\beta_{M, N} \leq \xi$. So $M \cap \alpha=M \cap \beta_{M, N}=N \cap \beta_{M, N}$. Thus $N \cap \alpha=N \cap \beta_{M, N}$. Therefore $\min (N \backslash \alpha)=\min \left(N \backslash \beta_{M, N}\right)$, which is in $R_{M}(N)$ and hence in $\Gamma$, giving a contradiction.

So indeed $\xi$ is not in $S$. Next we claim that for all $K \in A_{1}, \min (K \backslash \alpha)>\xi$. Consider $K \in A_{1}$ and let $\sigma=\min (K \backslash \alpha)$. Then $\sigma \in \Gamma$, and therefore $\sigma \neq \xi$. Suppose for a contradiction that $\sigma<\xi$. Then $\xi=\min (M \backslash \sigma)$. So if $\beta_{M, K} \leq \sigma$, 
then $\xi$ is in $R_{K}(M)$ and hence in $\Gamma$, which is false. Therefore $\sigma<\beta_{M, K}$. In particular, $\alpha<\beta_{M, K}$. But $\sup (K \cap \alpha)<\alpha=\sup (M \cap \alpha)$; so it must be the case that $K \cap \beta_{M, K} \in S k(M)$. This is impossible as $\sigma \in\left(K \cap \beta_{M, K}\right) \backslash M$. This proves that $\xi<\min (K \backslash \alpha)$.

Using Lemma 4.6, choose $\gamma$ in $M \cap S \cap \alpha$ which is larger than any ordinal below $\alpha$ which appears in a pair of $x_{p}$, and larger than $\sup (N \cap \alpha)$ for all $N$ in $A_{0} \cup A_{1}$. Let $r=\left(x_{p} \cup\{\langle\gamma, \xi\rangle\}, A_{p}\right)$. We claim that $r$ is a condition. This will contradict that $p$ forces that $\alpha$ is a limit point of $\dot{D}$, finishing the proof.

(1) Let $\langle\tau, \pi\rangle$ be in $x_{p}$. If either of $\tau$ or $\pi$ is below $\alpha$, then they are both below $\gamma$, and therefore $[\tau, \pi] \cap[\gamma, \xi]=\emptyset$. Otherwise they are both strictly above $\alpha$. We claim that $\tau>\xi$, which implies that the intervals are disjoint. If not, then $\alpha<\tau \leq \xi$. Since $\tau$ is in $S$ and $\xi$ is not, $\tau<\xi$. So $\tau$ is not in $M$. But then $\min (M \backslash \tau)=\xi$ is in $\Gamma$, which is false.

(2) is immediate. $(3,4)$ Let $K$ be in $A_{p}$. If $K$ is in $A_{0}$, then $K \cap[\gamma, \xi]=\emptyset$ and $\min (K \backslash \gamma)$ does not exist. If $K$ is in $A_{1}$, then again $K \cap[\gamma, \xi]=\emptyset$, and $\min (K \backslash \gamma)=\min (K \backslash \alpha)$ is in $\Gamma$ and hence in $S$. Suppose that $K$ is in $A_{2}$. Then $\alpha$ is a common limit point of $M$ and $K$, so $\alpha<\beta_{M, K}$. By the $\in$-minimality of $M$, $\gamma \in K$. If $\xi<\beta_{M, K}$, then again by the $\in$-minimality of $M$ we have that $\xi \in K$ and we are done. Otherwise $\alpha<\beta_{M, K}<\xi$. So $\xi=\min \left(M \backslash \beta_{M, K}\right)$ is in $R_{K}(M)$ and hence in $\Gamma$, which is a contradiction.

\section{REFERENCES}

[1] S.D. Friedman. Forcing with finite conditions. In Set Theory: Centre de Recerca Matemàtica, Barcelona, 2003-2004, Trends in Mathematics, pages 285-295. Birkhäuser Verlag, 2006.

[2] J. Krueger. Forcing with adequate sets of models as side conditions. To appear.

[3] W. Mitchell. $I\left[\omega_{2}\right]$ can be the nonstationary ideal on $\operatorname{Cof}\left(\omega_{1}\right)$. Trans. Amer. Math. Soc., $361(2): 561601,2009$.

[4] I. Neeman. Forcing with sequences of models of two types. To appear.

Department of Mathematics, University of North Texas, 1155 Union Circle \#311430, Denton, TX 76203

E-mail address: jkrueger@unt.edu 